\title{
Isolation and antioxidant activity of flavonoids from Holarrhena floribunda (G.don) leaves
}

\author{
Jelili A. Badmus¹, Okobi E. Ekpo1, Fanie Rautenbach², Jeanine L. Marnewick², \\ Ahmed A. Hussein ${ }^{3}$ and Donavon C. Hiss ${ }^{\mathbb{1}}$ \\ 1Department of Medical Biosciences, University of the Western Cape, New Life Sciences Building, Private Bag X17 Bellville, Cape Town 7535, \\ South Africa; ${ }^{2}$ Oxidative Stress Research Centre, Institute of Biomedical and Microbial Biotechnology, Faculty of Health and Wellness Sciences, \\ Cape Peninsula University of Technology, Cape Town South Africa; ${ }^{3}$ Department of Chemistry, University of the Western Cape, Chemical Sci- \\ ences Building, Private Bag X17 Bellville, Cape Town 7535, South Africa
}

\begin{abstract}
Bioactive polyphenolics are ubiquitously present in plants and may play an important role in the prevention and management of certain human diseases. Three known flavonoids viz Kaemperol-3-O-rutinoside (1), quercetin-3-O-glucoside (2) and kaemperol-3-O-glucoside (3) and inseparable mixture (1:1) of quercetin-3-Oglucose/galactose (4) were isolated, and identified for the first time from Holarrhena floribunda. The antioxidant capacity using the ORAC, FRAP and TEAC assays and inhibition of lipid peroxidation were measured for isolated flavonoids. The result showed that compounds 2 and 4 showed significantly increased ORAC, TEAC, and FRAP activities with low pro-oxidant potential as well as improved lipid peroxidation inhibition levels when compared to compounds 1 and 3. The most active compounds were found to be flavonoids with a quercetin basic structure. These results imply that the isolated flavonoid glycosides are responsible for the antioxidant activity of the plant leaves and it forms the scientific basis for its traditional usage.
\end{abstract}

Key words: polyphenolics, plants, Holarrhena floribunda, antioxidant, flavonoids

Received: 17 August, 2015; revised: 13 January, 2016; accepted: 11 February, 2016; available on-line: 12 May, 2016

\section{INTRODUCTION}

Flavonoids are low molecular weight, structurally related compounds with basic features of the 2-phenylbenzo- $\gamma$-prone nucleus consisting of two benzene rings linked through a heterocyclic pyran ring (Cushnie \& Lamb, 2005). The protective health benefits of natural flavonoids and its broad pharmacological activity cannot be overemphasized and has led to increased interest among scientists with bias in functional foods (Kahkonen et al., 1999). Flavonoids and other phenolic compounds with antioxidant activities are reputed to play a preventive role in the development of oxidative stress-related diseases like certain cancers (Kahkonen et al., 1999). Reactive oxygen species (ROS) produced during normal metabolism or induced by exogenous factors, have been implicated in the aetiology of several human diseases (Tapas et al., 2008). ROS is known to contribute to cellular aging, mutagenesis, carcinogenesis and coronary heart diseases (Sastre et al., 2000, Takabe et al., 2001, Kawanishi et al., 2001). The scavenging of ROS, metal chelating ability or induction of antioxidant en- zymes by the flavonoids are essential in preventing impending damage to important cellular components such as DNA, proteins and lipids (Suganya et al., 2007). Oxidation of lipids for example, leads to lipid peroxidation, which is a free-radical mediated propagation of oxidative insult to the polyunsaturated fatty acid component of cell membranes (Heim et al., 2002). The potential of the health benefit of these compounds for the prevention and therapeutic uses has led to the investigation and identification of a wide range of bioactive principles, that include mainly flavonoids and phenolic compounds in the plants (vegetables, fruits, leaves, seeds, cereal, roots, spices and herbs) (Suganya et al., 2007). The protective effects of flavonoids are attributed to their ability to transfer electrons, chelate metals, activate antioxidant enzymes, reduce alpha-tocopherol radicals and inhibit oxidases (Heim et al., 2002). Anti-inflammatory, anti-diarrhoeal, anti-ulcer, anti-viral, anti-allergic and vasodilatory actions have also been attributed to these phytochemicals (Proestos \& Komaitis, 2006). Previously, the methanolic extract and sub-fractions from the leaves of Holarrhena floribunda was reported to exhibit strong antioxidant activity (Badmus et al., 2010, Badmus et al., 2013). The present work sought to isolate and characterize the compounds responsible for the antioxidant activities.

\section{PLANT MATERIAL}

Holarrhena floribunda leaves were collected in Igbajo, Osun state, Nigeria during the raining season in July, 2011. It was identified and authenticated by the duo of Chukwuma, E.C and Ugbogu O.A at the Federal Research Institute of Nigeria (FRIN). Herbarium number FHI 109764 was issued at the Institute.

Preparation of methanolic leaf extract of Holarrhena floribunda. The leaves of Holarrbena floribunda were dried at room temperature. The dried leaves weighing $1.748 \mathrm{~kg}$ were extracted with $5.25 \mathrm{~L}$ methanol under stirring for $48 \mathrm{~h}$ (X 2). The filtrate was evaporated till dryness to give $380.70 \mathrm{~g}$ representing $21.78 \%$ yield (dry weight).The part of the dried total extract filtrate $(50 \mathrm{~g})$ was loaded into silica gel column eluted with a gradient

\footnotetext{
e-mail: dhiss@uwc.ac.za
}

Abbreviations: ABTS, 2,2'azabis-(3-ethyl benzothiozoline-6-sulfonate); AAPH, 2,2'-azobis (2-aminopropane); DCM, dichloromethane; EGCG, Epigallocatechin gallate; FRAP, ferric reducing antioxidant power; $\mathrm{MEOH}$, methanol; ORAC, oxygen radical absorption capacity; ROS, reactive oxygen species; TE, Trolox equivalent; TEAC, Trolox equivalent antioxidant capacity 
mixture of ethyl acetate and methanol. The ratio of ethyl acetate: methanol used was 9:1, 8:2, 6:4 and 4:6, respectively. A total of 51 eluents (500 $\mathrm{ml}$ each) was collected and pooled together according to their TLC profile to 18 main fractions.

Sub-fractions 13 and 14 from the main total extract were shown to contain flavonoids as indicated by TLC and selected for further purification. Both fractions (13 \& 14) were subjected to silica gel column eluted with dichloromethane:methanol (DCM: $\mathrm{MEOH}$ ) mixture 1\% to $15 \%$. The sub-fractions obtained were purified on Sephadex column using 100\% ethanol as mobile phase and finally high performance liquid chromatography (HPLC) using different ratios of acetonitrile and water to yield three pure compounds (1-3) and inseparable mixture (1:1) (4).

Oxygen radical absorption capacity (ORAC). ORAC generates both qualitative and quantitative measures which present fast and slow acting antioxidant activity of a test compound (DeLange \& Glazer, 1989). Briefly, $12 \mu \mathrm{l}$ of test sample $(0.1,1,10$ and $100 \mu \mathrm{g} /$ $\mathrm{ml}$ ) was combined with $138 \mu \mathrm{l}$ of the fluorescein working solution. The reaction was started by the addition of $50 \mu \mathrm{l}$ reactive species in 96-well plate. The absorbance was measured with a Fluoroskan spectrum plate reader with excitation/emission wavelengths set at 485/530 nm at $37^{\circ} \mathrm{C}$ against a reagent blank prepared with phosphate buffer. The method measures the antioxidant scavenging capacity of thermal decomposition generated by (1) peroxyl radical of 2,2'-azobis (2-aminopropane) dihydro-

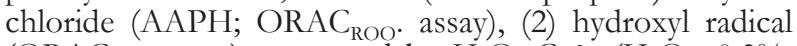
(ORAC $\mathrm{OH}$. assay), generated by $\mathrm{H}_{2} \mathrm{O}_{2}-\mathrm{Cu}^{2+}\left(\mathrm{H}_{2} \mathrm{O}_{2}, 0.3 \%\right.$; $\mathrm{Cu}^{2+}\left[\right.$ as $\left.\mathrm{CuSO}_{4}\right], 18 \mu \mathrm{M}$ ), or (3) $\mathrm{Cu}^{2+}\left[\right.$ as $\left.\mathrm{CuSO}_{4}\right], 18 \mu \mathrm{M}$ as a transition metal oxidant at $37^{\circ} \mathrm{C}$. ORAC values were expressed as micromole of Trolox equivalents (TE) per milligram dry weight ( $\mu \mathrm{M} \mathrm{TE} / \mathrm{mg} \mathrm{DW})$ of test sample, except when $\mathrm{Cu}^{2+}$ (without $\mathrm{H}_{2} \mathrm{O}_{2}$ ) was used as an oxidant in the assay. In the presence of $\mathrm{Cu}^{2+}$ without $\mathrm{H}_{2} \mathrm{O}_{2}$, test samples acted as prooxidants rather than antioxidants in the ORAC assay. The copper-initiated prooxidant activity was calculated using $\left[\left(\right.\right.$ Area $_{\text {Blank }}-$ Area $\left._{\text {Sample }}\right) /$ Area $\left._{\text {Blank }}\right] \times 100$ and expressed as prooxidant units; one unit equals the prooxidant activity that reduces the area under the fluorescein decay curve by $1 \%$ in the ORAC assay (Cao et al., 1997).

Ferric reducing antioxidant power (FRAP). FRAP measures a single electron transfer reaction from the antioxidant molecule to the oxidant. The change in absorbance value of either oxidant or antioxidant is a measure of reducing capability of the antioxidant (Ou et al., 2002).

A working FRAP reagent was prepared in accordance with previously described method (Benzie and Strain, 1999). The Samples (100 $\mu \mathrm{l}$; $[0.1,1,10,100 \mu \mathrm{g} / \mathrm{ml}])$ were prepared in triplicate followed by the addition of 3 $\mathrm{ml}$ freshly prepared FRAP reagent. The reaction mixture was incubated at $37^{\circ} \mathrm{C}$ for $4 \mathrm{~min}$ and absorbance read at $593 \mathrm{~nm}$ against the blank prepared with distilled water. L-Ascorbic acid was used as standard and the results were expressed as micromole ascorbic acid equivalent per milligram dry weight ( $\mu \mathrm{M} \mathrm{AAE} / \mathrm{mg} \mathrm{DW}$ ) of the test samples.

Trolox equivalent antioxidant capacity (TEAC). The TEAC assay estimates inhibition of radical cation production by the antioxidant in the sample. The concentration of antioxidant in the sample is inversely proportional to the absorbance of the radical cation produced by 2,2'-azo-bis-(3-ethyl benzothiozoline-6-sulfonate) (ABTS) (Gupta et al., 2009). TEAC assay is a useful assay for tracking down unknown antioxidants in a complex mixture (Arts et al., 2004). The assay was evaluated in accordance with previously described method (Re et al., 1999). Briefly, $25 \mu$ of test sample (0.1, $1,10,100 \mu \mathrm{g} / \mathrm{ml})$ was added to $300 \mu \mathrm{l}$ of ABTS $(1 \mathrm{ml}$ of ABTS in $20 \mathrm{ml}$ ethanol) in triplicate. The reaction mixture was incubated for $30 \mathrm{~min}$. Absorbance of the reaction was measured with a Multiskan spectrum plate reader at a wavelength of $734 \mathrm{~nm}$ at $25^{\circ} \mathrm{C}$ against the blank prepared with ethanol. Results were expressed as micromole Trolox equivalents per milligram dry weight $(\mu \mathrm{M} \mathrm{TE} / \mathrm{mgDW})$ of test samples.

Inhibition of $\mathrm{Fe}(\mathrm{II})$-induced microsomal lipid peroxidation. Inhibition of $\mathrm{Fe}(\mathrm{II})$-induced microsomal lipid peroxidation was determined by the method described by Snijman and co-workers (Snijman et al., 2009). Liver microsomes were prepared from male Fischer 344 rats. Microsomes were purified from the liver S9 fraction using a Sepharose 2B column as previously described (Gelderblom et al., 1984). The absorbance was read at $532 \mathrm{~nm}$. The percentage inhibition of sample relative to control was calculated by the equation below;

$\left[\left(\mathrm{A}_{\text {control }}-\mathrm{A}_{\text {sample }}\right) / \mathrm{A}_{\text {control }}\right] \times 100$.

Where $A_{\text {control }}$ and $A_{\text {sample }}$ refer to the absorbance of reacting mixture without the sample and the absorbance in the presence of sample respectively.

Statistical analyses. Data are expressed as means \pm S.D. of experiments performed in triplicate. The values were analyzed by Two-Way ANOVA, followed by Tukey's multiple comparison tests using GraphPad Prism software version 6 for Windows (GraphPad Software, La Jolla California USA, www.graphpad.com). A $P$ value of less than 0.05 was considered significant.

\section{RESULTS}

\section{Isolation and characterization of flavonoids from Holarrhena floribunda leaves}

TLC screening of different fractions from the methanolic leaf extract of Holarrbena floribunda, showed that sub-fraction 13 and 14 were rich in flavonoids, which were submitted for chromatographic purification using the combination of silica gel and sephadex column and HPLC. Compound 1 yielded $17.6 \mathrm{mg}$ while 2,3 and 4 (inseparable mixture (1:1) of quercetin-3-O-glucose/ galactose) gave $12.3,17.3$ and $14.72 \mathrm{mg}$, respectively (Fig. 1). The compounds were fully identified based on $1 \mathrm{D}$ and $2 \mathrm{D}$ NMR spectra. The data were compared with previously published study (Gudej, 2003; Sikorska \& Matlawska, 2000; Nowak \& Wolbis, 2002). These flavonoids were further subjected to antioxidant capacity test, including ORAC, FRAP, TEAC and lipid peroxidation inhibition.

\section{Evaluation of oxygen radical absorbance capacity}

The antioxidant evaluation of flavonoid-rich extracts using the ORAC assay in the presence of AAPH, $\mathrm{Cu}^{2+}$
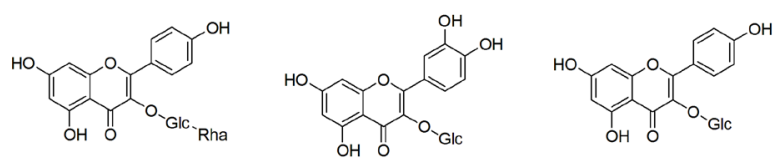

kaemperol-3-O-rutinoside (1)

quercetin-3-O-glucoside (2)

kaemperol-3-O- glucoside (3)

Figure 1. The structure of flavonoids isolated from the Holarrhena floribunda leaves. 


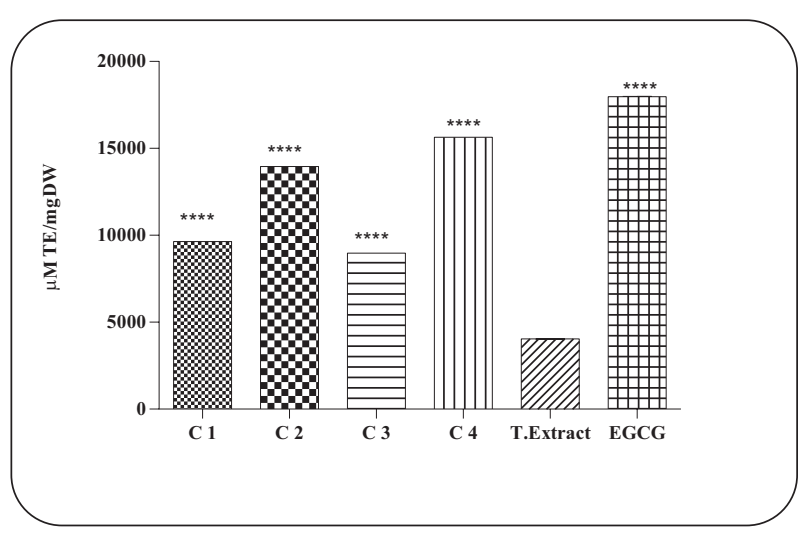

Figure 2. The ORAC values of isolated flavonoids, total extract and the standard (EGCG) using AAPH as an oxidant.

Data are expressed as mean \pm S.D. $\mu \mathrm{M}$ TE/mgDW. ${ }^{* * * *} P<0.0001$ when compared to T.Extract (Total extract). Note that the standard deviation is very low and as such is not showing in the figure.

$\mathrm{H}_{2} \mathrm{O}_{2}$ and $\mathrm{Cu}^{2+}$ showed that the isolated flavonoids and total extract have remarkable ORAC activities against the oxidants AAPH and $\mathrm{Cu}^{2+}-\mathrm{H}_{2} \mathrm{O}_{2}$ as shown in Figs. 2 and 3 below. However, Compounds 2 and 4 were significantly $(P<0.05)$ higher compared with compounds $\mathbf{1}, \mathbf{3}$ and the total extract in the AAPH and $\mathrm{Cu}^{2+}-\mathrm{H}_{2} \mathrm{O}_{2}$ systems. Figure 4 shows the percentage pro-oxidant ability of the compounds in the presence of $\mathrm{Cu}^{2+}$. The result (Fig. 4) showed that none of the isolated compounds and the total extract acted as a pro-oxidant in the presence of the $\mathrm{Cu}^{2+}$.

\section{Evaluation of ferric reducing antioxidant power}

The ability of compounds to reduce $\mathrm{Fe}^{3+}$ to $\mathrm{Fe}^{2+}$ was evaluated using the established FRAP method. Figure 5 below shows the ability of each compound and the total extract to reduce $\mathrm{Fe}^{3+}$ to $\mathrm{Fe}^{2+}$ calculated as micromole ascorbic acid equivalent per milligram dry weight ( $\mu \mathrm{mol} \mathrm{AAE} / \mathrm{mgDW})$. The present investigation showed that compounds 1 and $\mathbf{3}$ have significantly lower FRAP activity $(P<0.0001)$ when compared with compounds 2 , 4 and the methanolic extract with high activity (1561.37, 1527.63 and $850.06 \mu \mathrm{mol} \mathrm{AAE} / \mathrm{mgDW}$ respectively). The standard (EGCG; $4754 \mu \mathrm{mol}$ AAE/mgDW) was significantly higher than the isolated compounds and the total extract.

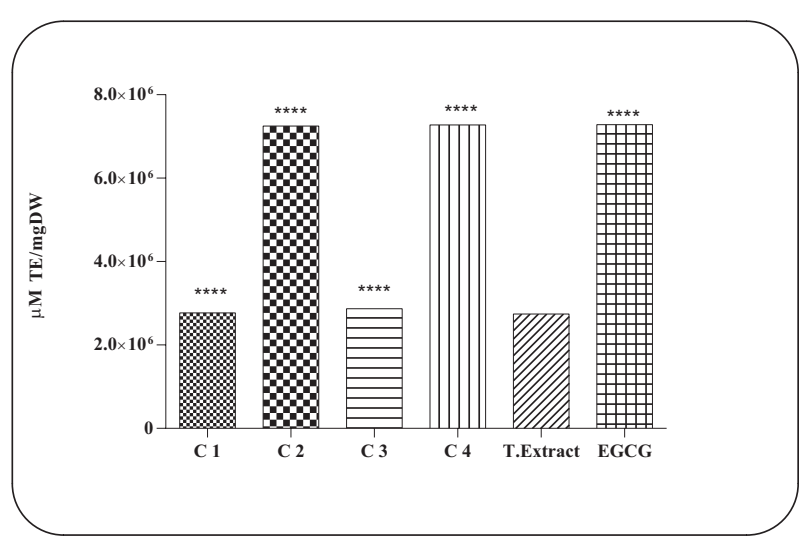

Figure 3. The ORAC values of isolated flavonoids, total extract and the standard (EGCG).

$\mathrm{Cu}^{2+}-\mathrm{H}_{2} \mathrm{O}_{2}$ was used to generate $\mathrm{OH}$ radical as an oxidant. Data are expressed as mean \pm S.D. $\mu \mathrm{M}$ TE/mgDW. ${ }^{* * *} P<0.0001$ when compared to the T.Extract (total extract).

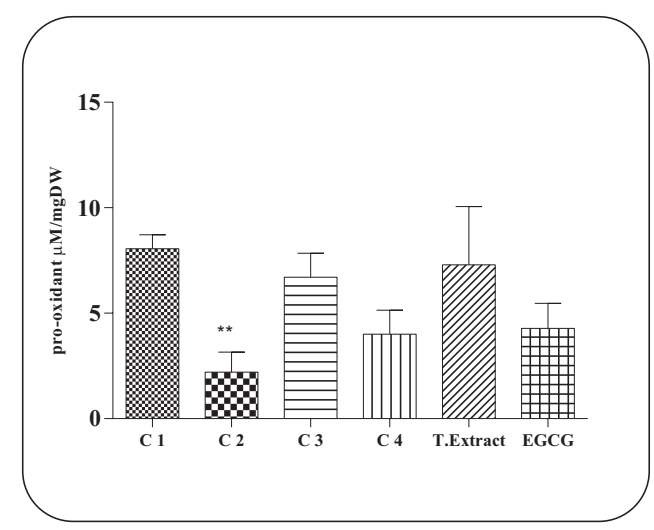

Figure 4. The percentage of pro-oxidant induction in relation to untreated control in the presence of $\mathrm{Cu}^{2+}$ evaluated using ORAC assay.

Data are expressed as mean \pm S.D. pro-oxidant $\mu \mathrm{M} / \mathrm{mgDW}$. ${ }^{*} P<0.05$ when compared to the T.Extract (total extract).

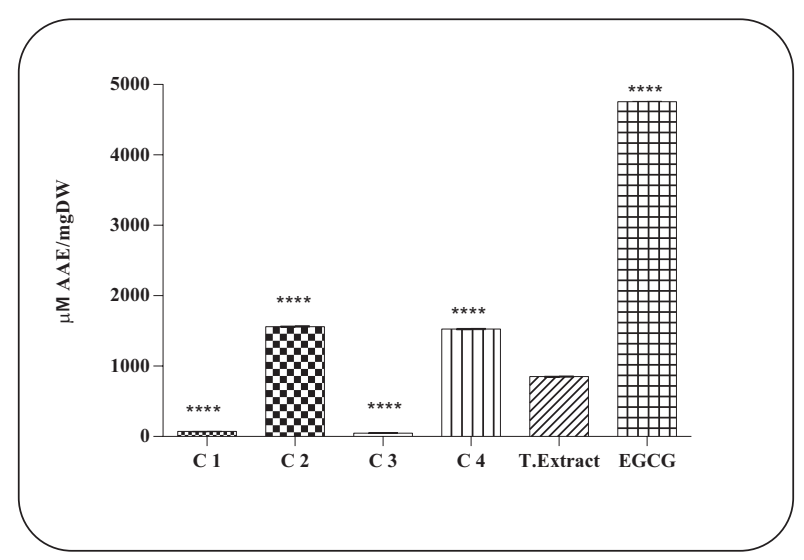

Figure 5. Ferric reducing antioxidant power assay (FRAP) of isolated compounds, total extract and the standard (EGCG).

Data are expressed as mean \pm S.D. $\mu$ M AAE/mgDW. ${ }^{* * *} \boldsymbol{P}<0.0001$ when compared to the T.Extract (total extract).

\section{Trolox equivalent antioxidant capacity (TEAC)}

Trolox equivalent antioxidant capacity of compounds and the total extract of Holarrhena floribunda are presented in Fig. 6. The TEAC assay involves evaluation of the quenching potential of antioxidants in the presence of

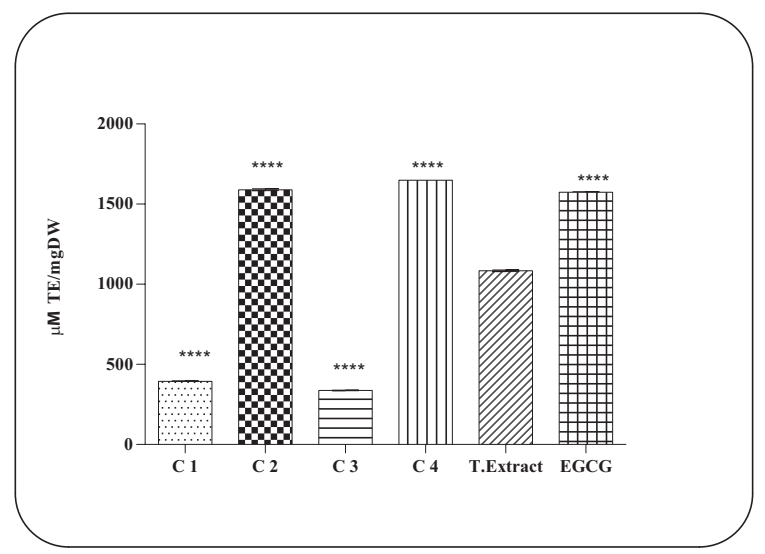

Figure 6. Trolox equivalent capacity (TEAC) of the isolated flavonoids and the total extract in $\mu$ mole trolox equivalent per gram dry weight.

Data are expressed as mean \pm .S.D. $\mu \mathrm{M} \mathrm{TE} / \mathrm{mgDW}$. ${ }^{* * *} P<0.0001$ when compared to the T.Extract (total extract). 
long-lived radical cation chromophore 2,2' azinobis-(3ethylbenzothiazoline-6-sulphonate) ABTS.+. The result of the TEAC assay follows the same manner as observed in the FRAP assay above. Compound 2 shows (1649.4 $\mu \mathrm{mol}$ TE/mgDW)>compound 4 (1589.9 $\mu \mathrm{mol}$ TE/ mgDW $)>$ EGCG $(1573.5 \mu \mathrm{mol} \mathrm{TE} / \mathrm{mgDW})>$ Total extract $(1084.4 \mu \mathrm{mol} \mathrm{TE} / \mathrm{mgDW})>$ compound 1 (394.8 $\mu \mathrm{mol} \mathrm{TE} / \mathrm{mgDW})>$ compound $\mathbf{3}$ (337.5 $\mu \mathrm{mol} \mathrm{TE} /$ mgDW). The results showed that the TEAC values for compounds 2, 4, Total extract and EGCG were significantly $(P<0.0001)$ higher when compared with compounds 1 and 3 .

\section{Inhibition of Fe-induced lipid peroxidation in rat liver microsome}

The effects of the compounds and the total extract on the induced-lipid peroxidation were evaluated in S9 rat liver fraction. The compounds 2, 4 and the extract showed dose dependent inhibition of lipid peroxidation, while dose dependent inhibitions were not observed in compounds 1 and 3 . The $\mathrm{IC}_{50}$ values of the results were calculated using the Prism 6 statistical software. The $\mathrm{IC}_{50}$ values (Table 1 ) showed that compound 2 has a $10.4 \mu \mathrm{g} / \mathrm{ml}$ while compounds 4 and the extract have 9.8 and $7.2 \mu \mathrm{g} / \mathrm{ml}$, respectively and $\mathrm{IC}_{50}$ values for compounds 1 and 3 could not be determined because they do not show dose dependent inhibition of lipid peroxidation. There is, however, no significant difference in the $\mathrm{IC}_{50}$ values among the compound 2, 4 and the extract.

\section{DISCUSSION}

Dietary antioxidants with potential for therapeutic and prevention use have been a major focus of research in recent years. Isolation and characterization of bioactive compounds from plants with health-maintaining and disease-preventing properties have led to the isolation and identification of an array of compounds among which include flavonoids (Suganya et al., 2007). The present work isolated flavonoids by subjecting the methanolic

Table 1. Inhibition of Fe-induced lipid peroxidation by isolated compounds and the total extract in rat liver microsome.

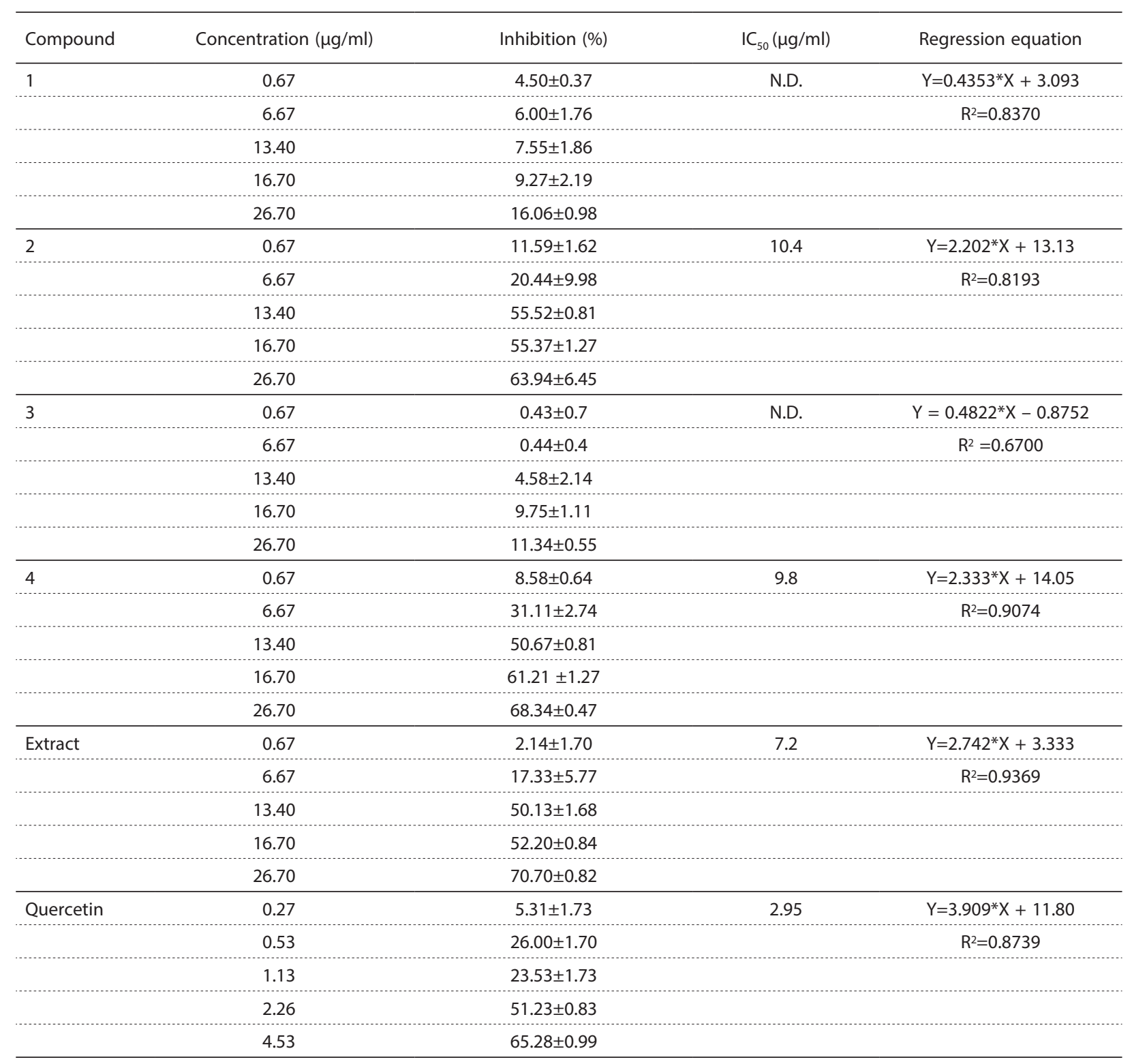


leaf fractions of Holarrhena floribunda to different chromatography techniques. The flavonoids were isolated, purified using HPLC, identified with NMR spectroscopy and data compared with that of the literature. Three pure compounds and an inseparable mixture of two compounds were isolated and identified. The compounds showed a dull spot under UV lights, which indicate the nature of flavonoids.

However, further to the isolation of flavonoids from the leaves, isolated flavonoids were subjected to antioxidant evaluation using ORAC, FRAP, TEAC and the in vitro inhibition of lipid peroxidation. The ORAC assay takes into account both inhibition time and the degree of inhibition into a single assay by considering area under the curve (Cao et al., 1995). In this study, in addition to the commonly used AAPH as an oxidant, $\mathrm{Cu}^{2+}-\mathrm{H}_{2} \mathrm{O}_{2}$ and $\mathrm{Cu}^{2+}$ alone were used as a source of oxidant. $\mathrm{Cu}^{2+}-\mathrm{H}_{2} \mathrm{O}_{2}$ is used to mimic the in vivo generation of oxidant as $\mathrm{H}_{2} \mathrm{O}_{2}$ and transition metal are available in vivo and are frequently used in vitro to induce oxidative damage to proteins and nucleic acids (Cao et al., 1997; Parthasarathy et al., 1989; Sato et al., 1992). In the presence of a transition metal like $\mathrm{Cu}^{2+}$ alone, it is believed that flavonoids act as a pro-oxidant (CaO et al., 1997). The $\mathrm{Cu}^{2+}$ was used in this study to evaluate pro-oxidative potential of the isolated flavonoids. Evaluation of the antioxidant capacity of the isolated compounds using the ORAC peroxyl radical $\left(\mathrm{ORAC}_{\mathrm{ROO}}\right.$ ) assay showed a high degree of antioxidant capacity for all the isolated compounds. However, compounds 2 and 4 showed significantly higher activities compared with the compounds 1 and 3. The ORAC $\mathrm{OH}_{\mathrm{OH}}$ activity of these compounds also followed a similar pattern to what is obtained in $\mathrm{ORAC}_{\mathrm{ROO}}$. activities. The ORAC $\mathrm{OH}_{\text {. }}$ activities of the flavonoids were several folds higher than what was obtained for ORAC $C_{\text {ROO. }}$ This could be that the flavonoids isolated were more specific to and aimed at $\mathrm{OH}$. radical protection. Chen et al. in their structural-activity relationships concluded that phenolic hydroxyls in flavonoids, hydroxyl groups in A and B rings (ortho-dihydroxyl groups in $\mathrm{A}$ and $\mathrm{B}$ rings), are important to the hydroxyl radical scavenging activity of flavonoids (Chen et al., 2002). Inversely however, the pro-oxidant activities using $\mathrm{Cu}^{2+}$ in the ORAC assay showed that compounds $\mathbf{1}$ and $\mathbf{3}$ were more prone to behave like prooxidants in the presence of the metal than the compounds 2 and 4 . This is not unexpected because the previous reports have correlated the number of flavonoids' OH group substitution to its ORAC activity (Rice-Evans et al., 1996). The observed results can be related to the basic structure of the flavonoids as compounds 2 and 4 have quercetin as their basic structure, while 1 and 3 have kaempferol. Cao et al.

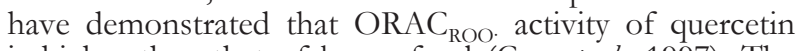
is higher than that of kaempferol (Cao et al., 1997). The pro-oxidant activity can also be related to the number of substituted $\mathrm{OH}$ groups in the flavonoids as it showed in this study that the lower the number of $\mathrm{OH}$ groups the higher the pro-oxidation capacity in the presence of $\mathrm{Cu}^{2+}$.

The importance of substitutions such as OH-group, methoxy group and O-dihydroxy in the B-ring are related to the high antioxidant activity of the flavonoids using a FRAP assay (Firuzi et al., 2005). The results of the FRAP assay of this study show that $\mathrm{OH}$ group substitution in the compounds 2 and 4 are responsible for the significant antioxidant activity compared to compounds 1 and 3 . In the same vain, similar activities were shown by the isolated flavonoids when subjected to TEAC antioxidant assay. The result implies that the activities of these flavonoids remarkably correlate with the 3'-OH group on the B-ring of flavonoids basic structure which is the only different between quercetin and kaempferol flavonoids structure. The strong antioxidant activity of the extract, however, implies that the isolated compounds 2 and 4 are responsible for the observed antioxidant activity in the total extract although they are not complementary.

Endogenous and exogenous factors such as metabolism, chemicals and ionizing radiations are linked to the induction of free radicals in biological tissues (Iqbal et al., 2003). Iron is known to be involved in the generation of reactive oxygen species (ROS) in vivo which may attack lipids to form damaged products (Imlay \& Linn, 1988; Aruoma et al., 1989). Lipid peroxidation is an important product of reaction between free radicals and lipids (D'Souza \& Prabhu, 2006). The ability of an agent to protect lipids from oxidative damage by ROS can be related to its antioxidant ability.

Iron-induced lipid peroxidation is a reliable biological marker of cellular oxidative stress (Dargel, 1992). The result, showed that compounds $\mathbf{1}$ and $\mathbf{3}$ did not exhibit significant inhibition of lipid peroxidation, as the values did not reach up to $50 \%$ inhibition in the concentration used while compounds 2, 4 and total extract showed non-significant $\mathrm{IC}_{50}$ values when compared to each other. The lipid peroxidation inhibitory activities of flavonoids are shown to be related to the number of hydroxyl group, substitution of the hydroxyl group, catechol moiety on the B-ring and double bonds between carbon 2 and 3 of the C-ring (Cholbi et al., 1991, Mora et al., 1990). The presence of a sugar moiety however affects the activity of the lipid peroxidation inhibition due to steric hindrance between the sugar and adjacent hydroxyl group likewise methoxy group (Cholbi et al., 1991). This can be applied to the results obtained in the present study when the inhibition of lipid peroxidation of the isolated glycosylated flavonoids was compared with the quercetin standard. The result showed that $\mathrm{IC}_{50}$ inhibition of peroxidation by the standard is about 3 folds lower than the isolated flavonoids. On the other hand, glycosylation of flavonoids increased the hydrophilicity and hence enhances bioavailability better than for the aglycone flavonoids (Kumar \& Pandey, 2013). However, despite lower activity of glycosylated flavonoids compared to their aglycone, bioavailability will be a determining factor of their bioactivity in vivo (Thilakarathna \& Rupasinghe, 2013). Enhancement of bioavailability will be an important factor in order to exert an eventual beneficial effect in vivo (Thilakarathna \& Rupasinghe, 2013). In addition, the results obtained in this study is in agreement with the number of hydroxyl group substitution on the B-ring as compounds 2 and 4 with hydroxyl group in B-ring showed effective dose dependent lipid peroxidation compared with compounds 1 and 3 .

In summary, the antioxidant activity of flavonoids, which involve neutralization of free radicals initiating oxidative-cascade of reactions or termination of the free radical chain reaction due to hydrogen donating property, can be related to their structures (Cao et al., 1997; Suganya et al., 2007). Compounds 2 and 4 have an orthodihydroxyl in the B-ring of the flavonoid skeleton (catechol) which plays an essential role in the antioxidant activity of flavonoids (Bors et al., 1990) and is responsible for the observed effects in those compounds. The antioxidant activity of flavonoids is known to depend largely on the functional groups attached to the nuclear structure (Heim et al., 2002). It is noteworthy to observe that in the battery of antioxidant assays done in this study, no significant differences could be shown when compar- 
ing compounds 2 and 4 and compounds 1 and 3 , which showed selective antioxidant activities only. This implies that the only common factor that is responsible for the observed differences in the antioxidant activities of the flavonoids in this study is ortho-dihydroxyl in the B-ring.

This work has lend scientific credence to the claim by the tradition that the leave of Holarrbena floribunda has medicinal values and also that the plant can be a source of natural antioxidant which is beneficial for maintaining the health status of humans.

\section{Acknowledgements}

The authors thank TETFund (Nigeria) for PhD travel scholarship granted to the first author. The work was sponsored, in part, by the National Research Foundation (NRF), South Africa.

\section{REFERENCES}

Arts MJ, Haenen GR, Voss HP, Bast A (2004) Antioxidant capacity of reaction products limits the applicability of the Trolox Equivalent Antioxidant Capacity (TEAC) assay. Food Chem Toxicol 42: 45-49. doi:10.1016/i.fct.2003.08.004.

Aruoma OI, Halliwell B, Gajewski E, Dizdaroglu M (1989) Damage to the bases in DNA induced by hydrogen peroxide and ferric ion chelates. J Biol Chem 264: 20509-20512.

Badmus JA, Odunola OA, Obuotor EM, Oyedapo OO (2010) Phytochemicals and in vitro antioxidant potentials of defatted methanolic extract of Holarrbena floribunda leaves. African J Biotecbnol 9: 340-346. doi: 10.5897/AJB09.734.

Badmus JA, Odunola OA, Yekeen TA et al. (2013) Evaluation of antioxidant, antimutagenic, and lipid peroxidation inhibitory activities of selected fractions of Holarrhena floribunda (G. Don) leaves. Acta Biochim Pol 60: 435-442.

Benzie IF, Strain JJ (1999) Ferric reducing/antioxidant power assay: direct measure of total antioxidant activity of biological fluids and modified version for simultaneous measurement of total antioxidant power and ascorbic acid concentration. Methods Ensymol 299: 15-27.

Bors W, Heller W, Michel C, Saran M (1990) Flavonoids as antioxidants: Determination of radical-scavenging efficiencies. Methods Ensymol 186: 343-355. doi:10.1016/0076-6879(90)86128-I.

Cao G, Sofic E, Prior RL (1997) Antioxidant and prooxidant behavior of flavonoids: structure-activity relationships. Free Radic Biol Med 22: $749-760$.

Cao G, Verdon CP, Wu AH, Wang H, Prior RL (1995) Automated assay of oxygen radical absorbance capacity with the COBAS FARA II. Clin Chem 41: 1738-1744.

Chen JW, Zhu ZQ, Hu TX, Zhu DY (2002) Structure-activity relationship of natural flavonoids in hydroxyl radical-scavenging effects. Acta Pharmacol Sin 23: 667-672.

Cholbi MR, Paya M, Alcaraz MJ (1991) Inhibitory effects of phenolic compounds on CCl4-induced microsomal lipid peroxidation. Experientia 47: 195-199. doi: 10.1016/j.cbi.2012.04.003.

Cushnie TPT, Lamb AJ (2005) Antimicrobial activity of flavonoids. Int J Antimicrob Agents 26: 343-356. doi: 10.1016/j.ijantimicag.2005.09.002.

D'Souza HP, Prabhu HR (2006) In vitro inhibition of lipid peroxidation in fish by turmeric (Curcuma longa). Indian J Clin Biocbem 21: 1381341. doi: $10.1007 /$ BF02912929.

Dargel R (1992) Lipid peroxidation - a common pathogenetic mechanism? Exp Toxicol Pathol 44: 169-181.

DeLange RJ, Glazer AN (1989) Phycoerythrin fluorescence-based assay for peroxy radicals: a screen for biologically relevant protective agents. Anal Biochem 177: 300-306. doi: 10.1016/00032697(89)90056-0

Firuzi O, Lacanna A, Petrucci R, Marrosu G, Saso L (2005) Evaluation of the antioxidant activity of flavonoids by "ferric reducing antioxidant power" assay and cyclic voltammetry. Biochim Biophys Acta 1721: 174-184. doi: 10.1016/j.bbagen.2004.11.001.

Gelderblom WC, Thiel PG, van der Merwe KJ (1984) Metabolic activation and deactivation of fusarin $\mathrm{C}$, a mutagen produced by Fusar- ium moniliforme. Biochem Pharmacol 33: 1601-1603. doi: 10.1016/00062952(84)90280-6.

Gudej J (2003) Kaempferol and quercetin glycosides from Rubus idaeus L. leaves. Acta Pol Pharm 60: 313-315.

Gupta R, Sharma M, Lakshmy R, Prabhakaran D, Reddy KS (2009) Improved method of total antioxidant assay. Indian J Biochem Biophys 46: $126-129$.

Heim KE, Tagliaferro AR, Bobilya DJ (2002) Flavonoid antioxidants: chemistry, metabolism and structure-activity relationships. J Nutr Biochem 13: 572-584. doi:10.1016/S0955-2863(02)00208-5.

Imlay JA, Linn S (1988) DNA damage and oxygen radical toxicity. Science 240: 1302-1309.

Iqbal M, Okazaki Y, Okada S (2003) In vitro curcumin modulates ferric nitrilotriacetate (Fe-NTA) and hydrogen peroxide ( $\mathrm{H} 2 \mathrm{O} 2)-$ induced peroxidation of microsomal membrane lipids and DNA damage. Teratog Carcinog Mutagen (Suppl 1): 151-160.

Kahkonen MP, Hopia AI, Vuorela HJ, et al. (1999) Antioxidant activity of plant extracts containing phenolic compounds. J Agric Food Chem 47: 3954-3962. doi:10.1021/jf9901461.

Kawanishi S, Hiraku Y, Oikawa S (2001) Mechanism of guanine-specific DNA damage by oxidative stress and its role in carcinogenesis and aging. Mutat Res 488: 65-76. doi:10.1016/S1383-5742(00)00059-4.

Kumar S, Pandey AK (2013) Chemistry and biological activities of flavonoids: an overview. Scientific World Journal 2013: 162750. doi:10.1155/2013/162750.

Mora A, Paya M, Rios JL, Alcaraz MJ (1990) Structure-activity relationships of polymethoxyflavones and other flavonoids as inhibitors of non-enzymic lipid peroxidation. Biochem Pharmacol 40: 793-797. doi: 10.1016/0006-2952(90)90317-E.

Nowak S, Wolbis M (2002) Flavonoids from some species of genus Scopolia Jacq. Acta Pol Pharm 59: 275-280.

Ou B, Huang D, Hampsch-Woodill M, Flanagan JA, Deemer EK (2002) Analysis of antioxidant activities of common vegetables employing oxygen radical absorbance capacity (ORAC) and ferric reducing antioxidant power (FRAP) assays: a comparative study. J Agric Food Chem 50: 3122-3128. doi: 10.1021/jf0116606.

Parthasarathy S, Wieland E, Steinberg D (1989) A role for endothelial cell lipoxygenase in the oxidative modification of low density lipoprotein. Proc Natl Acad Sci USA 86: 1046-1050. doi: 10.1073/ pnas.86.3.1046.

Proestos C, Komaitis M (2006) Ultrasonically assisted extraction of phenolic compounds from aromatic plants: comparison with conventional extraction techniques. J Food Qual 29: 567-582.

Re R, Pellegrini N, Proteggente A, Pannala A, Yang M, Rice-Evans C (1999) Antioxidant activity applying an improved ABTS radical cation decolorization assay. Free Radic Biol Med 26: 1231-1237. doi:10.1016/S0891-5849(98)00315-3.

Rice-Evans CA, Miller NJ, Paganga G (1996) Structure-antioxidant activity relationships of flavonoids and phenolic acids. Free Radic Biol Med 20: 933-956. doi: 10.1016/0891-5849(95)02227-9.

Sastre J, Pallardo FV, Vina J (2000) Mitochondrial oxidative stress plays a key role in aging and apoptosis. IUBMB Life 49: 427-435. doi: $10.1080 / 152165400410281$

Sato K, Akaike T, Kohno M, Ando M, Maeda H (1992) Hydroxyl radical production by $\mathrm{H}_{2} \mathrm{O}_{2}$ plus $\mathrm{Cu}, \mathrm{Zn}$-superoxide dismutase reflects the activity of free copper released from the oxidatively damaged enzyme. J Biol Chem 267: 25371-25377.

Sikorska M, Matlawska I (2000) Quercetin and its glycosides in the flowers of Asclepias syriaca L. Acta Pol Pharm 57: 321-324.

Snijman PW, Joubert E, Ferreira D, et al. (2009) Antioxidant activity of the dihydrochalcones Aspalathin and Nothofagin and their corresponding flavones in relation to other Rooibos (Aspalatbus linearis) Flavonoids, Epigallocatechin Gallate, and Trolox. J Agric Food Chem 57: 6678-6684. doi: 10.1021/jf901417k.

Suganya T, Fumio I, Siriporn O (2007) Antioxidants active principles isolated from Psidium guajava grown inThailand. Sci Pharm 75: 179193. doi:10.3797/scipharm.2007.

Takabe W, Niki E, Uchida K, Yamada S, Satoh K, Noguchi N (2001) Oxidative stress promotes the development of transformation: involvement of a potent mutagenic lipid peroxidation product, acrolein. Carcinogenesis 22: 935-941.

Tapas AR, Sakarkar DM, Kakde RB (2008) Flavonoids as nutraceuticals: A review. Tropical J Pharm Res 7: 1089-1099. doi.org/10.4314/ tipr.v7i3.14693.

Thilakarathna SH, Rupasinghe HP (2013) Flavonoid bioavailability and attempts for bioavailability enhancement. Nutrients 5: 3367-3387. doi: $10.3390 /$ nu5093367. 